\title{
New Applications of Sălăgean and Ruscheweyh Operators for Obtaining Fuzzy Differential Subordinations
}

\author{
Alina Alb Lupaş *,+(i) and Georgia Irina Oros ${ }^{+}(\mathbb{D}$ \\ Department of Mathematics and Computer Science, University of Oradea, 1 Universitatii Street, \\ 410087 Oradea, Romania; georgia_oros_ro@yahoo.co.uk \\ * Correspondence: alblupas@gmail.com \\ + These authors contributed equally to this work.
}

check for updates

Citation: Alb Lupaş, A.; Oros, G.I. New Applications of Sălăgean and Ruscheweyh Operators for Obtaining Fuzzy Differential Subordinations. Mathematics 2021, 9, 2000. https:/ / doi.org/10.3390/math9162000

Academic Editor: Alfonso Mateos Caballero

Received: 22 July 2021

Accepted: 16 August 2021

Published: 21 August 2021

Publisher's Note: MDPI stays neutral with regard to jurisdictional claims in published maps and institutional affiliations.

Copyright: (c) 2021 by the authors. Licensee MDPI, Basel, Switzerland. This article is an open access article distributed under the terms and conditions of the Creative Commons Attribution (CC BY) license (https:/ / creativecommons.org/licenses/by/ $4.0 /)$.
Abstract: The present paper deals with notions from the field of complex analysis which have been adapted to fuzzy sets theory, namely, the part dealing with geometric function theory. Several fuzzy differential subordinations are established regarding the operator $L_{\alpha}^{m}$, given by $L_{\alpha}^{m}: \mathcal{A}_{n} \rightarrow \mathcal{A}_{n}$, $L_{\alpha}^{m} f(z)=(1-\alpha) R^{m} f(z)+\alpha S^{m} f(z)$, where $\mathcal{A}_{n}=\left\{f \in \mathcal{H}(U), f(z)=z+a_{n+1} z^{n+1}+\ldots, z \in U\right\}$ is the subclass of normalized holomorphic functions and the operators $R^{m} f(z)$ and $S^{m} f(z)$ are Ruscheweyh and Sălăgean differential operator, respectively. Using the operator $L_{\alpha}^{m}$, a certain fuzzy class of analytic functions denoted by $S L_{\mathcal{F}}^{m}(\delta, \alpha)$ is defined in the open unit disc. Interesting results related to this class are obtained using the concept of fuzzy differential subordination. Examples are also given for pointing out applications of the theoretical results contained in the original theorems and corollaries.

Keywords: fuzzy differential subordination; convex function; fuzzy best dominant; differential operator

\section{Introduction}

Fuzzy sets theory epic started in 1965 when Lotfi A. Zadeh published the paper "Fuzzy Sets" [1], received with distrust at first but currently cited by over 95,000 papers. Mathematicians have been constantly concerned with adapting fuzzy sets theory to different branches of mathematics, and many such connections have been made. The beautiful review paper published in 2017 [2] is a tribute to Lotfi A. Zadeh's contribution to the scientific world and shows the evolution of the notion of fuzzy set in time and its numerous connections with different topics of mathematics, science, and technique. Another great review article published as part of this Special Issue dedicated to the Centenary of the Birth of Lotfi A. Zadeh [3] gives further details on the development of fuzzy sets theory and highlights the contributions of Professor I. Dzitac who has had Lotfi A. Zadeh as mentor. In 2008, he edited a volume [4], tying his name to that of Lotfi A. Zadeh for posterity.

The first applications of fuzzy sets theory in the part of complex analysis studying analytic functions of one complex variable were marked by the introduction of the concept of fuzzy subordination in 2011 [5]. The study was continued, and the notion of fuzzy differential subordination was introduced in 2012 [6]. All the aspects of the classical theory of differential subordination which are synthesized in the monograph published in 2000 [7] by the same authors who have introduced the notion in 1978 [8] and 1981 [9] were then adapted in light of the connection to fuzzy sets theory. At some point, fuzzy differential subordinations began to be studied in connection with different operators with many applications in geometric function theory as it can be seen in the first papers published starting with 2013 [10-12]. The topic is of obvious interest at this time, a fact proved by the numerous papers published in the last 2 years, of which we mention only a few [13-16].

In this paper, fuzzy differential subordinations will be obtained using the differential operator defined and studied in several aspects in $[17,18]$. 
The basic notions used for conducting the study are denoted as previously established in literature.

Let $U=\{z \in \mathbb{C}:|z|<1\}$ and denote by $\mathcal{H}(U)$ the class of holomorphic functions in the unit disc $\mathcal{U}$. Let $\mathcal{A}_{n}=\left\{f \in \mathcal{H}(U): f(z)=z+a_{n+1} z^{n+1}+\ldots, z \in U\right\}$ be the subclass of normalized holomorphic functions writing $\mathcal{A}_{1}$ as $\mathcal{A}$. When $a \in \mathbb{C}$ and $n \in \mathbb{N}^{*}$, denote by $\mathcal{H}[a, n]=\left\{f \in \mathcal{H}(U): f(z)=a+a_{n} z^{n}+a_{n+1} z^{n+1}+\ldots, z \in U\right\}$ writing $\mathcal{H}_{0}=\mathcal{H}[0,1]$. The class of convex functions is obtained for $\alpha=0$ when $0<\alpha<1$ the class denoted by $\mathcal{K}(\alpha)=\left\{f \in \mathcal{A}: \operatorname{Re} \frac{z f^{\prime \prime}(z)}{f^{\prime}(z)}+1>\alpha, z \in U\right\}$ contains convex functions of order $\alpha$.

The definitions necessary for using the concept of fuzzy differential subordinations introduced in previously published cited papers are next reminded.

Definition 1 ([19]). A pair $\left(A, F_{A}\right)$, where $F_{A}: X \rightarrow[0,1]$ and $A=\left\{x \in X: 0<F_{A}(x) \leq 1\right\}$ is called fuzzy subset of $X$. The set $A$ is called the support of the fuzzy set $\left(A, F_{A}\right)$ and $F_{A}$ is called the membership function of the fuzzy set $\left(A, F_{A}\right)$. One can also denote $A=\operatorname{supp}\left(A, F_{A}\right)$.

Remark 1. If $A \subset X$, then $F_{A}(x)=\left\{\begin{array}{l}1, \text { if } x \in A \\ 0, \text { if } x \notin A\end{array}\right.$.

For a fuzzy subset, the real number 0 represents the smallest membership degree of a certain $x \in X$ to $A$ and the real number 1 represents the biggest membership degree of a certain $x \in X$ to $A$.

The empty set $\varnothing \subset X$ is characterized by $F_{\varnothing}(x)=0, x \in X$, and the total set $X$ is characterized by $F_{X}(x)=1, x \in X$.

Definition 2 ([5]). Let $D \subset \mathbb{C}, z_{0} \in D$ be a fixed point and let the functions $f, g \in \mathcal{H}(D)$. The function $f$ is said to be fuzzy subordinate to $g$ and write $f \prec_{\mathcal{F}} g$ or $f(z) \prec_{\mathcal{F}} g(z)$, if the conditions are satisfied:

(1) $f\left(z_{0}\right)=g\left(z_{0}\right)$,

(2) $F_{f(D)} f(z) \leq F_{g(D)} g(z), z \in D$.

Definition 3 ([6] (Definition 2.2)). Let $\psi: \mathbb{C}^{3} \times U \rightarrow \mathbb{C}$ and h univalent in $U$, with $\psi(a, 0 ; 0)=$ $h(0)=a$. If $p$ is analytic in $U$, with $p(0)=a$ and satisfies the (second-order) fuzzy differential subordination

$$
F_{\psi\left(\mathbb{C}^{3} \times U\right)} \psi\left(p(z), z p^{\prime}(z), z^{2} p^{\prime \prime}(z) ; z\right) \leq F_{h(U)} h(z), \quad z \in U,
$$

then $p$ is called a fuzzy solution of the fuzzy differential subordination. The univalent function $q$ is called a fuzzy dominant of the fuzzy solutions of the fuzzy differential subordination, or more simple a fuzzy dominant, if $F_{p(U)} p(z) \leq F_{q(U)} q(z), z \in U$, for all $p$ satisfying (1). A fuzzy dominant $\tilde{q}$ that satisfies $F_{\tilde{q}(U)} \tilde{q}(z) \leq F_{q(U)} q(z), z \in U$, for all fuzzy dominants $q$ of $(1)$ is said to be the fuzzy best dominant of (1).

Lemma 1 ([7] (Corollary 2.6g.2, p. 66)). Let $h \in \mathcal{A}_{n}$ and

$$
L[f](z)=F(z)=\frac{1}{n z^{\frac{1}{n}}} \int_{0}^{z} h(t) t^{\frac{1}{n}-1} d t, z \in U .
$$

If

$$
\operatorname{Re}\left(\frac{z h^{\prime \prime}(z)}{h^{\prime}(z)}+1\right)>-\frac{1}{2}, z \in U
$$

then $L(f)=F \in \mathcal{K}$. 
Lemma 2 ([20]). Let $h$ be a convex function with $h(0)=a$, and let $\gamma \in \mathbb{C}^{*}$ be a complex number with Re $\gamma \geq 0$. If $p \in \mathcal{H}[a, n]$ with $p(0)=a, \psi: \mathbb{C}^{2} \times U \rightarrow \mathbb{C}, \psi\left(p(z), z p^{\prime}(z) ; z\right)=$ $p(z)+\frac{1}{\gamma} z p^{\prime}(z)$ an analytic function in $U$ and

$$
F_{\psi\left(\mathbb{C}^{2} \times U\right)}\left(p(z)+\frac{1}{\gamma} z p^{\prime}(z)\right) \leq F_{h(U)} h(z), \text { i.e., } p(z)+\frac{1}{\gamma} z p^{\prime}(z) \prec \mathcal{F} h(z), \quad z \in U,
$$

then

$$
F_{p(U)} p(z) \leq F_{g(U)} g(z) \leq F_{h(U)} h(z) \text {, i.e., } p(z) \prec \mathcal{F} g(z) \prec \mathcal{F} h(z), \quad z \in U,
$$

where $g(z)=\frac{\gamma}{n z^{\gamma / n}} \int_{0}^{z} h(t) t^{\gamma / n-1} d t, z \in U$. The function $q$ is convex and is the fuzzy best dominant.

Lemma 3 ([20]). Let $g$ be a convex function in $U$ and let $h(z)=g(z)+n \alpha z g^{\prime}(z), z \in U$, where $\alpha>0$ and $n$ is a positive integer.

If $p(z)=g(0)+p_{n} z^{n}+p_{n+1} z^{n+1}+\ldots, z \in U$, is holomorphic in $U$ and

$$
F_{p(U)}\left(p(z)+\alpha z p^{\prime}(z)\right) \leq F_{h(U)} h(z) \text {, i.e., } p(z)+\alpha z p^{\prime}(z) \prec \mathcal{F} h(z), \quad z \in U,
$$

then

$$
F_{p(U)} p(z) \leq F_{g(U)} g(z), \text { i.e., } p(z) \prec \mathcal{F} g(z), \quad z \in U,
$$

and this result is sharp.

Sălăgean and Ruscheweyh differential operators are well known in geometric function theory for the nice results obtained by implementing them in the studies. Their definitions and basic properties are given in the next two definitions and remarks.

Definition 4 (Sălăgean [21]). For $f \in \mathcal{A}_{n}, m, n \in \mathbb{N}$, the operator $S^{m}$ is defined by $S^{m}: \mathcal{A}_{n} \rightarrow \mathcal{A}_{n}$,

$$
\begin{aligned}
S^{0} f(z) & =f(z) \\
S^{1} f(z)= & z f^{\prime}(z) \\
& \cdots \\
S^{m+1} f(z)= & z\left(S^{m} f(z)\right)^{\prime}, z \in U .
\end{aligned}
$$

Remark 2. If $f \in \mathcal{A}_{n}, f(z)=z+\sum_{j=n+1}^{\infty} a_{j} z^{j}$, then $S^{m} f(z)=z+\sum_{j=n+1}^{\infty} j^{m} a_{j} z^{j}, z \in U$.

Definition 5 (Ruscheweyh [22]). For $f \in \mathcal{A}_{n}, m, n \in \mathbb{N}$, the operator $R^{m}$ is defined by $R^{m}$ : $\mathcal{A}_{n} \rightarrow \mathcal{A}_{n}$

$$
\begin{aligned}
R^{0} f(z) & =f(z) \\
R^{1} f(z)= & z f^{\prime}(z) \\
& \cdots \\
(m+1) R^{m+1} f(z) & =z\left(R^{m} f(z)\right)^{\prime}+m R^{m} f(z), z \in U .
\end{aligned}
$$

Remark 3. If $f \in \mathcal{A}_{n}, f(z)=z+\sum_{j=n+1}^{\infty} a_{j} z^{j}$, then $R^{m} f(z)=z+\sum_{j=n+1}^{\infty} C_{m+j-1}^{m} a_{j} z^{j}, z \in U$.

The next definition shows the operator used for obtaining the original results of this paper, defined in a previously published paper. Two remarks regarding it are also listed.

Definition 6 ([17]). Let $\alpha \geq 0, m, n \in \mathbb{N}$. Denote by $L_{\alpha}^{m}$ the operator given by $L_{\alpha}^{m}: \mathcal{A}_{n} \rightarrow \mathcal{A}_{n}$,

$$
L_{\alpha}^{m} f(z)=(1-\alpha) R^{m} f(z)+\alpha S^{m} f(z), \quad z \in U
$$


Remark 4. $L_{\alpha}^{m}$ is a linear operator and if $f \in \mathcal{A}_{n}, f(z)=z+\sum_{j=n+1}^{\infty} a_{j} z^{j}$, then $L_{\alpha}^{m} f(z)=$ $z+\sum_{j=n+1}^{\infty}\left(\alpha j^{m}+(1-\alpha) C_{m+j-1}^{m}\right) a_{j} z^{j}, z \in U$.

Remark 5. For $\alpha=0, L_{0}^{m} f(z)=R^{m} f(z), z \in U$, and for $\alpha=1, L_{1}^{m} f(z)=S^{m} f(z), z \in U$.

For $m=0, L_{\alpha}^{0} f(z)=(1-\alpha) R^{0} f(z)+\alpha S^{0} f(z)=f(z)=R^{0} f(z)=S^{0} f(z), z \in U$, and for $m=1, L_{\alpha}^{1} f(z)=(1-\alpha) R^{1} f(z)+\alpha S^{1} f(z)=z f^{\prime}(z)=R^{1} f(z)=S^{1} f(z), z \in U$.

Definition 7 ([11]). Let $f(D)=\sup p\left(f(D), F_{f(D)}\right)=\left\{z \in D: 0<F_{f(D)} f(z) \leq 1\right\}$, where $F_{f(D)}$. is the membership function of the fuzzy set $f(D)$ associated to the function $f$.

The membership function of the fuzzy set $(\mu f)(D)$ associated to the function $\mu f$ coincides with the membership function of the fuzzy set $f(D)$ associated to the function $f$, i.e., $F_{(\mu f)(D)}((\mu f)(z))=F_{f(D)} f(z), z \in D$.

The membership function of the fuzzy set $(f+g)(D)$ associated to the function $f+g$ coincide with the half of the sum of the membership functions of the fuzzy sets $f(D)$, respectively $g(D)$, associated to the function $f$, respectively $g$, i.e., $F_{(f+g)(D)}((f+g)(z))=\frac{F_{f(D)} f(z)+F_{g(D)} g(z)}{2}$, $z \in D$.

Remark 6. As $0<F_{f(D)} f(z) \leq 1$ and $0<F_{g(D)} g(z) \leq 1$, it is evident that $0<F_{(f+g)(D)}((f+g)(z)) \leq 1, z \in D$.

\section{Main Results}

First, a new fuzzy class of analytic functions is defined using the operator given by Definition 6.

Definition 8. The fuzzy class denoted $S L_{\mathcal{F}}^{m}(\delta, \alpha)$ contains all functions $f \in \mathcal{A}_{n}$ which satisfy the fuzzy inequality

$$
F_{\left(L_{\alpha}^{m} f\right)^{\prime}(U)}\left(L_{\alpha}^{m} f(z)\right)^{\prime}>\delta, \quad z \in U,
$$

when $\delta \in(0,1], \alpha \geq 0$ and $m, n \in \mathbb{N}$.

The first result for this class is related to its convexity.

Theorem 1. The set $S L_{\mathcal{F}}^{m}(\delta, \alpha)$ is convex.

Proof. Consider the functions

$$
f_{j}(z)=z+\sum_{j=n+1}^{\infty} a_{j k} z^{j} \in S L_{\mathcal{F}}^{m}(\delta, \alpha), \quad k=1,2, \quad z \in U .
$$

For obtaining the required conclusion, the function

$$
h(z)=\eta_{1} f_{1}(z)+\eta_{2} f_{2}(z)
$$

must be part of the class $S L_{\mathcal{F}}^{m}(\delta, \alpha)$, with $\eta_{1}$ and $\eta_{2}$ non-negative such that $\eta_{1}+\eta_{2}=1$.

We next show that $h \in S L_{\mathcal{F}}^{m}(\delta, \alpha)$,

$h^{\prime}(z)=\left(\mu_{1} f_{1}+\mu_{2} f_{2}\right)^{\prime}(z)=\mu_{1} f_{1}^{\prime}(z)+\mu_{2} f_{2}^{\prime}(z), z \in U$, and

$\left(L_{\alpha}^{m} h(z)\right)^{\prime}=\left(L_{\alpha}^{m}\left(\mu_{1} f_{1}+\mu_{2} f_{2}\right)(z)\right)^{\prime}=\mu_{1}\left(L_{\alpha}^{m} f_{1}(z)\right)^{\prime}+\mu_{2}\left(L_{\alpha}^{m} f_{2}(z)\right)^{\prime}$.

From Definition 7 we obtain that

$$
\begin{aligned}
& F_{\left(L_{\alpha}^{m} h\right)^{\prime}(U)}\left(L_{\alpha}^{m} h(z)\right)^{\prime}=F_{\left(L_{\alpha}^{m}\left(\mu_{1} f_{1}+\mu_{2} f_{2}\right)\right)^{\prime}(U)}\left(L_{\alpha}^{m}\left(\mu_{1} f_{1}+\mu_{2} f_{2}\right)(z)\right)^{\prime}= \\
& F_{\left(L_{\alpha}^{m}\left(\mu_{1} f_{1}+\mu_{2} f_{2}\right)\right)^{\prime}(U)}\left(\mu_{1}\left(L_{\alpha}^{m} f_{1}(z)\right)^{\prime}+\mu_{2}\left(L_{\alpha}^{m} f_{2}(z)\right)^{\prime}\right)= \\
& \frac{F_{\left(\mu_{1} L_{\alpha}^{m} f_{1}\right)^{\prime}(U)}\left(\mu_{1}\left(L_{\alpha}^{m} f_{1}(z)\right)^{\prime}\right)+F_{\left(\mu_{2} L_{\alpha}^{m} f_{2}\right)^{\prime}(U)}\left(\mu_{2}\left(L_{\alpha}^{m} f_{2}(z)\right)^{\prime}\right)}{2}=\frac{F_{\left(L_{\alpha}^{m} f_{1}\right)^{\prime}(U)}\left(L_{\alpha}^{m} f_{1}(z)\right)^{\prime}+F_{\left(L_{\alpha}^{m} f_{2}\right)^{\prime}(U)}\left(L_{\alpha}^{m} f_{2}(z)\right)^{\prime}}{2} .
\end{aligned}
$$


As $f_{1}, f_{2} \in S L_{\mathcal{F}}^{m}(\delta, \alpha)$ we have $\delta<F_{\left(L_{\alpha}^{m} f_{1}\right)^{\prime}(U)}\left(L_{\alpha}^{m} f_{1}(z)\right)^{\prime} \leq 1$ and $\delta<F_{\left(L_{\alpha}^{m} f_{2}\right)^{\prime}(U)}$ $\left(L_{\alpha}^{m} f_{2}(z)\right)^{\prime} \leq 1, z \in U$. Therefore, $\delta<\frac{F_{\left(L_{\alpha}^{m} f_{1}\right)^{\prime}(U)}\left(L_{\alpha}^{m} f_{1}(z)\right)^{\prime}+F_{\left(L_{\alpha}^{m} f_{2}\right)^{\prime}(U)}\left(L_{\alpha}^{m} f_{2}(z)\right)^{\prime}}{2} \leq 1$ and we obtain that $\delta<F_{\left(L_{\alpha}^{m} h\right)^{\prime}(U)}\left(L_{\alpha}^{m} h(z)\right)^{\prime} \leq 1$, which means that $h \in S L_{\mathcal{F}}^{m}(\delta, \alpha)$ and $S L_{\mathcal{F}}^{m}(\delta, \alpha)$ is convex.

A fuzzy subordination result is given in the next theorem and a related example follows.

Theorem 2. Considering the convex function in $U$ denoted by $g$ and defining $h(z)=g(z)+$ $\frac{1}{c+2} z g^{\prime}(z)$, with $c>0, z \in U$, if $f \in S L_{\mathcal{F}}^{m}(\delta, \alpha)$ and $G(z)=I_{\mathcal{c}}(f)(z)=\frac{c+2}{z^{c+1}} \int_{0}^{z} t^{c} f(t) d t, z \in U$, then the fuzzy differential subordination

$$
F_{\left(L_{\alpha}^{m} f\right)^{\prime}(U)}\left(L_{\alpha}^{m} f(z)\right)^{\prime} \leq F_{h(U)} h(z), \text { i.e., }\left(L_{\alpha}^{m} f(z)\right)^{\prime} \prec_{\mathcal{F}} h(z), \quad z \in U,
$$

implies

$$
F_{\left(L_{\alpha}^{m} G\right)^{\prime}(U)}\left(L_{\alpha}^{m} G(z)\right)^{\prime} \leq F_{g(U)} g(z), \text { i.e., }\left(L_{\alpha}^{m} G(z)\right)^{\prime} \prec \mathcal{F} g(z), \quad z \in U,
$$

and this result is sharp.

Proof. Using the definition of function $G(z)$, we obtain

$$
z^{c+1} G(z)=(c+2) \int_{0}^{z} t^{c} f(t) d t
$$

Differentiating (5) with respect to $z$, we have $(c+1) G(z)+z G^{\prime}(z)=(c+2) f(z)$ and

$$
(c+1) L_{\alpha}^{m} G(z)+z\left(L_{\alpha}^{m} G(z)\right)^{\prime}=(c+2) L_{\alpha}^{m} f(z), \quad z \in U .
$$

Differentiating (6) we have

$$
\left(L_{\alpha}^{m} G(z)\right)^{\prime}+\frac{1}{c+2} z\left(L_{\alpha}^{m} G(z)\right)^{\prime \prime}=\left(L_{\alpha}^{m} f(z)\right)^{\prime}, \quad z \in U .
$$

Using (7), the fuzzy differential subordination (4) becomes

$$
F_{L_{\alpha}^{m} G(U)}\left(\left(L_{\alpha}^{m} G(z)\right)^{\prime}+\frac{1}{c+2} z\left(L_{\alpha}^{m} G(z)\right)^{\prime \prime}\right) \leq F_{g(U)}\left(g(z)+\frac{1}{c+2} z g^{\prime}(z)\right) .
$$

If we denote

$$
p(z)=\left(L_{\alpha}^{m} G(z)\right)^{\prime}, \quad z \in U,
$$

then $p \in \mathcal{H}[1, n]$.

Replacing (9) in (8) we obtain

$$
F_{p(U)}\left(p(z)+\frac{1}{c+2} z p^{\prime}(z)\right) \leq F_{g(U)}\left(g(z)+\frac{1}{c+2} z g^{\prime}(z)\right), \quad z \in U .
$$

Using Lemma 3, we have

$$
F_{p(U)} p(z) \leq F_{g(U)} g(z), \quad z \in U, \quad \text { i.e., } \quad F_{\left(L_{\alpha}^{m} G\right)^{\prime}(U)}\left(L_{\alpha}^{m} G(z)\right)^{\prime} \leq F_{g(U)} g(z), \quad z \in U,
$$

and $g$ is the best dominant. We have obtained

$$
\left(L_{\alpha}^{m} G(z)\right)^{\prime} \prec \mathcal{F} g(z), \quad z \in U .
$$

Example 1. If $f \in S L_{\mathcal{F}}^{1}\left(1, \frac{1}{2}\right)$, then $f^{\prime}(z)+z f^{\prime \prime}(z) \prec \mathcal{F} \frac{3-2 z}{3(1-z)^{2}}$ implies $G^{\prime}(z)+z G^{\prime \prime}(z) \prec \mathcal{F}$ $\frac{1}{1-z}$, where $G(z)=\frac{3}{z^{2}} \int_{0}^{z} t f(t) d t$. 
Several fuzzy subordination results are contained in the next theorems and corollaries. Some are followed by examples.

Theorem 3. Let $h(z)=\frac{1+(2 \beta-1) z}{1+z}, \beta \in[0,1)$ and $c>0$. If $\alpha \geq 0, m \in \mathbb{N}$ and $I_{\mathcal{c}}(f)(z)=$ $\frac{c+2}{z^{c+1}} \int_{0}^{z} t^{c} f(t) d t, z \in U$, then

$$
I_{\mathcal{C}}\left[S L_{\mathcal{F}}^{m}(\beta, \alpha)\right] \subset S L_{\mathcal{F}}^{m}\left(\beta^{*}, \alpha\right),
$$

where $\beta^{*}=2 \delta-1+\frac{(c+2)(2-2 \delta)}{n} \int_{0}^{1} \frac{t \frac{c+2}{n}-1}{1+t} d t$.

Proof. As function $h$ given in the theorem is convex, we can use the same arguments as in the proof of Theorem 2. Interpreting the hypothesis of Theorem 3, we read that

$$
F_{p(U)}\left(p(z)+\frac{1}{c+2} z p^{\prime}(z)\right) \leq f_{h(U)} h(z),
$$

where $p(z)$ is given by (9).

By applying Lemma 2, the following fuzzy inequality is obtained:

$F_{p(U)} p(z) \leq F_{g(U)} g(z) \leq F_{h(U)} h(z)$, i.e., $\quad F_{\left(L_{\alpha}^{m} G\right)^{\prime}(U)}\left(L_{\alpha}^{m} G(z)\right)^{\prime} \leq F_{g(U)} g(z) \leq F_{h(U)} h(z)$, where

$$
g(z)=\frac{c+2}{n z^{\frac{c+2}{n}}} \int_{0}^{z} t^{\frac{c+2}{n}-1} \frac{1+(2 \delta-1) t}{1+t} d t=(2 \delta-1)+\frac{(c+2)(2-2 \delta)}{n z^{\frac{c+2}{n}}} \int_{0}^{z} \frac{t^{\frac{c+2}{n}-1}}{1+t} d t
$$

Using the hypothesis of convexity for function $g$, it is known that $g(U)$ is symmetric with respect to the real axis and we can write

$$
F_{L_{\alpha}^{m} G(U)}\left(L_{\alpha}^{m} G(z)\right)^{\prime} \geq \min _{|z|=1} F_{g(U)} g(z)=F_{g(U)} g(1)
$$

and $\beta^{*}=g(1)=2 \delta-1+\frac{(c+2)(2-2 \delta)}{n} \int_{0}^{1} \frac{\frac{c+2}{n}-1}{1+t} d t$.

From (11) we deduce inclusion (10).

Theorem 4. Let $g$ be a convex function with $g(0)=1$ and define the function $h(z)=g(z)+$ $z g^{\prime}(z), z \in U$.

If a function $f \in \mathcal{A}_{n}$ satisfies

$$
F_{\left(L_{\alpha}^{m} f\right)^{\prime}(U)}\left(L_{\alpha}^{m} f(z)\right)^{\prime} \leq F_{h(U)} h(z), \text { i.e., }\left(L_{\alpha}^{m} f(z)\right)^{\prime} \prec \mathcal{F} h(z), \quad z \in U,
$$

for $\alpha \geq 0$ and $m, n \in \mathbb{N}$, then we obtain the fuzzy differential subordination

$$
F_{L_{\alpha}^{m} f(U)} \frac{L_{\alpha}^{m} f(z)}{z} \leq F_{g(U)} g(z), \text { i.e., } \frac{L_{\alpha}^{m} f(z)}{z} \prec_{\mathcal{F}} g(z), \quad z \in U,
$$

and this result is sharp.

Proof. Using Remark 4 concerning the operator $L_{\alpha}^{m}$, we can write

$$
L_{\alpha}^{m} f(z)=z+\sum_{j=n+1}^{\infty}\left(\alpha j^{m}+(1-\alpha) C_{m+j-1}^{m}\right) a_{j} z^{j}, \quad z \in U .
$$

$z \in U$.

Consider $p(z)=\frac{L_{\alpha}^{m} f(z)}{z}=\frac{z+\sum_{j=n+1}^{\infty}\left(\alpha j^{m}+(1-\alpha) C_{m+j-1}^{m}\right) a_{j} z^{j}}{z}=1+p_{n} z^{n}+p_{n+1} z^{n+1}+\ldots$,

We deduce that $p \in \mathcal{H}[1, n]$. 
Let $L_{\alpha}^{m} f(z)=z p(z)$, for $z \in U$. Differentiating the expression we obtain $\left(L_{\alpha}^{m} f(z)\right)^{\prime}=$ $p(z)+z p^{\prime}(z), z \in U$.

Using this result in (12), we can write

$$
F_{p(U)}\left(p(z)+z p^{\prime}(z)\right) \leq F_{h(U)} h(z)=F_{g(U)}\left(g(z)+z g^{\prime}(z)\right), \quad z \in U .
$$

We can now apply Lemma 3 and obtain

$$
F_{p(U)} p(z) \leq F_{g(U)} g(z), \quad z \in U, \quad \text { i.e., } \quad F_{\left(L_{\alpha}^{m} f\right)^{\prime}(U)} \frac{L_{\alpha}^{m} f(z)}{z} \leq F_{g(U)} g(z), \quad z \in U .
$$

Therefore,

$$
\frac{L_{\alpha}^{m} f(z)}{z} \prec_{\mathcal{F}} g(z), \quad z \in U,
$$

and this result is sharp.

Theorem 5. Let $h$ be a convex function of order $-\frac{1}{2}$ with $h(0)=1$. If a function $f \in \mathcal{A}_{n}$ satisfies

$$
F_{\left(L_{\alpha}^{m} f\right)^{\prime}(U)}\left(L_{\alpha}^{m} f(z)\right)^{\prime} \leq F_{h(U)} h(z), \text { i.e., }\left(L_{\alpha}^{m} f(z)\right)^{\prime} \prec \mathcal{F} h(z), \quad z \in U,
$$

for $\alpha \geq 0$ and $m, n \in \mathbb{N}$, then

$$
F_{L_{\alpha}^{m} f(U)} \frac{L_{\alpha}^{m} f(z)}{z} \leq F_{q(U)} q(z), \text { i.e., } \frac{L_{\alpha}^{m} f(z)}{z} \prec_{\mathcal{F}} q(z), \quad z \in U,
$$

where $q(z)=\frac{1}{n z^{\frac{1}{n}}} \int_{0}^{z} h(t) t^{\frac{1}{n}-1} d t$ is convex and is the fuzzy best dominant.

Proof. Let

$$
\begin{aligned}
p(z) & =\frac{L_{\alpha}^{m} f(z)}{z}=\frac{z+\sum_{j=n+1}^{\infty}\left(\alpha j^{m}+(1-\alpha) C_{m+j-1}^{m}\right) a_{j} z^{j}}{z} \\
& =1+\sum_{j=n+1}^{\infty}\left(\alpha j^{m}+(1-\alpha) C_{m+j-1}^{m}\right) a_{j} z^{j-1}=1+\sum_{j=n+1}^{\infty} p_{j} z^{j-1}, z \in U, p \in \mathcal{H}[1, n] .
\end{aligned}
$$

As $\operatorname{Re}\left(1+\frac{z h^{\prime \prime}(z)}{h^{\prime}(z)}\right)>-\frac{1}{2}, z \in U$, from Lemma 1 , we obtain that $q(z)=\frac{1}{n z^{\frac{1}{n}}} \int_{0}^{z} h(t) t^{\frac{1}{n}-1} d t$ is a convex function and verifies the differential equation associated to the fuzzy differential subordination (13) $q(z)+z q^{\prime}(z)=h(z)$, therefore it is the fuzzy best dominant.

Differentiating, we obtain $\left(L_{\alpha}^{m} f(z)\right)^{\prime}=p(z)+z p^{\prime}(z), z \in U$ and (13) becomes

$$
F_{p(U)}\left(p(z)+z p^{\prime}(z)\right) \leq F_{h(U)} h(z), \quad z \in U .
$$

Using Lemma 3, we have

$$
F_{p(U)} p(z) \leq F_{q(U)} q(z), \quad z \in U, \quad \text { i.e., } \quad F_{L_{\alpha}^{m} f(U)} \frac{L_{\alpha}^{m} f(z)}{z} \leq F_{q(U)} q(z), \quad z \in U .
$$

We have obtained that

$$
\frac{L_{\alpha}^{m} f(z)}{z} \prec_{\mathcal{F}} q(z), \quad z \in U
$$

Corollary 1. Let $h(z)=\frac{1+(2 \beta-1) z}{1+z}$ a convex function in $U, 0 \leq \beta<1$. If $\alpha \geq 0, m, n \in \mathbb{N}$, $f \in \mathcal{A}_{n}$ and verifies the fuzzy differential subordination

$$
F_{\left(L_{\alpha}^{m} f\right)^{\prime}(U)}\left(L_{\alpha}^{m} f(z)\right)^{\prime} \leq F_{h(U)} h(z), \text { i.e., }\left(L_{\alpha}^{m} f(z)\right)^{\prime} \prec \mathcal{F} h(z), \quad z \in U,
$$


then

$$
F_{L_{\alpha}^{m} f(U)} \frac{L_{\alpha}^{m} f(z)}{z} \leq F_{q(U)} q(z), \text { i.e., } \frac{L_{\alpha}^{m} f(z)}{z} \prec_{\mathcal{F}} q(z), \quad z \in U,
$$

where $q$ is given by $q(z)=2 \beta-1+\frac{2(1-\beta)}{n z^{\frac{1}{n}}} \int_{0}^{z} \frac{t^{\frac{1}{n}-1}}{1+t} d t, z \in U$. The function $q$ is convex and it is the fuzzy best dominant.

Proof. We have $h(z)=\frac{1+(2 \beta-1) z}{1+z}$ with $h(0)=1, h^{\prime}(z)=\frac{-2(1-\beta)}{(1+z)^{2}}$ and $h^{\prime \prime}(z)=\frac{4(1-\beta)}{(1+z)^{3}}$, therefore $\operatorname{Re}\left(\frac{z h^{\prime \prime}(z)}{h^{\prime}(z)}+1\right)=\operatorname{Re}\left(\frac{1-z}{1+z}\right)=\operatorname{Re}\left(\frac{1-\rho \cos \theta-i \rho \sin \theta}{1+\rho \cos \theta+i \rho \sin \theta}\right)=\frac{1-\rho^{2}}{1+2 \rho \cos \theta+\rho^{2}}>0>-\frac{1}{2}$.

Following the same steps as in the proof of Theorem 5 and considering $p(z)=\frac{L_{\alpha}^{m} f(z)}{z}$, the fuzzy differential subordination (14) becomes

$$
F_{L_{\alpha}^{n} f(U)}\left(p(z)+z p^{\prime}(z)\right) \leq F_{h(U)} h(z), \quad z \in U .
$$

By using Lemma 2 for $\gamma=1$, we have $F_{p(U)} p(z) \leq F_{q(U)} q(z)$, i.e.,

$$
F_{L_{\alpha}^{n} f(U)} \frac{L_{\alpha}^{m} f(z)}{z} \leq F_{q(U)} q(z)
$$

and

$$
\begin{gathered}
q(z)=\frac{1}{n z^{\frac{1}{n}}} \int_{0}^{z} h(t) t^{\frac{1}{n}-1} d t= \\
\frac{1}{n z^{\frac{1}{n}}} \int_{0}^{z} t^{\frac{1}{n}-1} \frac{1+(2 \beta-1) t}{1+t} d t=2 \beta-1+\frac{2(1-\beta)}{n z^{\frac{1}{n}}} \int_{0}^{z} \frac{t^{\frac{1}{n}-1}}{1+t} d t, \quad z \in U .
\end{gathered}
$$

Example 2. Let $h(z)=\frac{1-z}{1+z}$ with $h(0)=1, h^{\prime}(z)=\frac{-2}{(1+z)^{2}}$ and $h^{\prime \prime}(z)=\frac{4}{(1+z)^{3}}$.

As $\operatorname{Re}\left(\frac{z h^{\prime \prime}(z)}{h^{\prime}(z)}+1\right)=\operatorname{Re}\left(\frac{1-z}{1+z}\right)=\operatorname{Re}\left(\frac{1-\rho \cos \theta-i \rho \sin \theta}{1+\rho \cos \theta+i \rho \sin \theta}\right)=\frac{1-\rho^{2}}{1+2 \rho \cos \theta+\rho^{2}}>0>-\frac{1}{2}$, the function $h$ is convex in $U$.

Let $f(z)=z+z^{2}, z \in U$. For $n=1, m=1, \alpha=2$, we obtain $L_{2}^{1} f(z)=-R^{1} f(z)+$ $2 S^{1} f(z)=-z f^{\prime}(z)+2 z f^{\prime}(z)=z f^{\prime}(z)=z+2 z^{2}$. Then, $\left(L_{2}^{1} f(z)\right)^{\prime}=1+4 z$ and $\frac{L_{2}^{1} f(z)}{z}=$ $1+2 z$.

We have $q(z)=\frac{1}{z} \int_{0}^{z} \frac{1-t}{1+t} d t=-1+\frac{2 \ln (1+z)}{z}$.

Using Theorem 5 we obtain

$$
1+4 z \prec \mathcal{F} \frac{1-z}{1+z}, \quad z \in U,
$$

induces

$$
1+2 z \prec_{\mathcal{F}}-1+\frac{2 \ln (1+z)}{z}, \quad z \in U .
$$

Theorem 6. Define the function $h(z)=g(z)+z g^{\prime}(z), z \in U$, using $g$ a convex function in $U$ with $g(0)=1$. If a function $f \in \mathcal{A}_{n}$ satisfies

$$
F_{L_{\alpha}^{m} f(U)}\left(\frac{z L_{\alpha}^{m+1} f(z)}{L_{\alpha}^{m} f(z)}\right)^{\prime} \leq F_{h(U)} h(z), \text { i.e., }\left(\frac{z L_{\alpha}^{m+1} f(z)}{L_{\alpha}^{m} f(z)}\right)^{\prime} \prec_{\mathcal{F}} h(z), \quad z \in U
$$

for $\alpha \geq 0$ and $m, n \in \mathbb{N}$, then we obtain the sharp fuzzy differential subordination

$$
F_{L_{\alpha}^{m} f(U)} \frac{L_{\alpha}^{m+1} f(z)}{L_{\alpha}^{m} f(z)} \leq F_{g(U)} g(z), \text { i.e., } \frac{L_{\alpha}^{m+1} f(z)}{L_{\alpha}^{m} f(z)} \prec_{\mathcal{F}} g(z), \quad z \in U \text {. }
$$

Proof. For $f \in \mathcal{A}_{n}, f(z)=z+\sum_{j=n+1}^{\infty} a_{j} z^{j}$ we have 
$L_{\alpha}^{m} f(z)=z+\sum_{j=n+1}^{\infty}\left(\alpha j^{m}+(1-\alpha) C_{m+j-1}^{m}\right) a_{j} z^{j}, z \in U$.

Consider

$$
p(z)=\frac{L_{\alpha}^{m+1} f(z)}{L_{\alpha}^{m} f(z)}=\frac{z+\sum_{j=n+1}^{\infty}\left(\alpha j^{m+1}+(1-\alpha) C_{m+j}^{m+1}\right) a_{j} z^{j}}{z+\sum_{j=n+1}^{\infty}\left(\alpha j^{m}+(1-\alpha) C_{m+j-1}^{m}\right) a_{j} z^{j}} .
$$

We have $p^{\prime}(z)=\frac{\left(L_{\alpha}^{m+1} f(z)\right)^{\prime}}{L_{\alpha}^{m} f(z)}-p(z) \cdot \frac{\left(L_{\alpha}^{m} f(z)\right)^{\prime}}{L_{\alpha}^{m} f(z)}$ and we obtain $p(z)+z \cdot p^{\prime}(z)=$ $\left(\frac{z L_{\alpha}^{m+1} f(z)}{L_{\alpha}^{m} f(z)}\right)^{\prime}$.

Relation (15) becomes

$$
F_{p(U)}\left(p(z)+z p^{\prime}(z)\right) \leq F_{h(U)} h(z)=F_{g(U)}\left(g(z)+z g^{\prime}(z)\right), \quad z \in U .
$$

By using Lemma 3, we have

$$
F_{p(U)} p(z) \leq F_{g(U)} g(z), \quad z \in U, \quad \text { i.e., } \quad F_{L_{\alpha}^{m} f(U)} \frac{L_{\alpha}^{m+1} f(z)}{L_{\alpha}^{m} f(z)} \leq F_{g(U)} g(z), \quad z \in U .
$$

We obtain

$$
\frac{L_{\alpha}^{m+1} f(z)}{L_{\alpha}^{m} f(z)} \prec_{\mathcal{F}} g(z), \quad z \in U
$$

Theorem 7. Given a convex function $g$ with $g(0)=1$, define function $h(z)=g(z)+z g^{\prime}(z)$, $z \in U$.

If we take $\alpha \geq 0$ and $m, n \in \mathbb{N}$ and a function $f \in \mathcal{A}_{n}$ satisfying

$$
\begin{gathered}
F_{L_{\alpha}^{m} f(U)}\left(\left(L_{\alpha}^{m+1} f(z)\right)^{\prime}+\frac{(1-\alpha) m z\left(R^{m} f(z)\right)^{\prime \prime}}{m+1}\right) \leq F_{h(U)} h(z), \text { i.e., } \\
\left(L_{\alpha}^{m+1} f(z)\right)^{\prime}+\frac{(1-\alpha) m z\left(R^{m} f(z)\right)^{\prime \prime}}{m+1} \prec_{\mathcal{F}} h(z), \quad z \in U,
\end{gathered}
$$

then the sharp fuzzy differential subordination results

$$
F_{\left(L_{\alpha}^{m} f\right)^{\prime}(U)}\left[L_{\alpha}^{m} f(z)\right]^{\prime} \leq F_{g(U)} g(z) \text {, i.e., }\left[L_{\alpha}^{m} f(z)\right]^{\prime} \prec_{\mathcal{F}} g(z), \quad z \in U \text {. }
$$

Proof. Using the definition of operator $L_{\alpha}^{m}$, we get

$$
L_{\alpha}^{m+1} f(z)=(1-\alpha) R^{m+1} f(z)+\alpha S^{m+1} f(z), \quad z \in U .
$$

Using this result in (16), we obtain

$$
\begin{aligned}
& F_{L_{\alpha}^{m} f(U)}\left(\left((1-\alpha) R^{m+1} f(z)+\alpha S^{m+1} f(z)\right)^{\prime}+\frac{(1-\alpha) m z\left(R^{m} f(z)\right)^{\prime \prime}}{m+1}\right) \leq F_{h(U)} h(z), z \in U, \\
& F_{L_{\alpha}^{m} f(U)}\left((1-\alpha)\left(R^{m} f(z)\right)^{\prime}+\alpha\left(S^{m} f(z)\right)^{\prime}+z\left((1-\alpha)\left(R^{m} f(z)\right)^{\prime \prime}+\alpha\left(S^{m} f(z)\right)^{\prime \prime}\right)\right) \leq F_{h(U)} h(z), z \in U \text {. } \\
& \text { Let } \\
& p(z)=(1-\alpha)\left(R^{m} f(z)\right)^{\prime}+\alpha\left(S^{m} f(z)\right)^{\prime}=\left(L_{\alpha}^{m} f(z)\right)^{\prime} \\
& =1+\sum_{j=n+1}^{\infty}\left(\alpha j^{m+1}+(1-\alpha) j C_{m+j-1}^{m}\right) a_{j} z^{j-1}=1+p_{n} z^{n}+p_{n+1} z^{n+1}+\ldots
\end{aligned}
$$

We deduce that $p \in \mathcal{H}[1, n]$. 
Using the notation in (18), the fuzzy differential subordination becomes

$$
F_{p(U)}\left(p(z)+z p^{\prime}(z)\right) \leq F_{h(U)} h(z)=F_{g(U)}\left(g(z)+z g^{\prime}(z)\right) .
$$

By using Lemma 3, we have

$$
F_{p(U)} p(z) \leq F_{g(U)} g(z), \quad z \in U, \quad \text { i.e., } \quad F_{L_{\alpha}^{m} f(U)}\left(L_{\alpha}^{m} f(z)\right)^{\prime} \leq F_{g(U)} g(z), \quad z \in U,
$$

and this result is sharp.

Theorem 8. Let $h$ be a convex function of order $-\frac{1}{2}$ which satisfies $h(0)=1$. If a function $f \in \mathcal{A}_{n}$ satisfies

$$
\begin{gathered}
F_{L_{\alpha}^{m} f(U)}\left(\left(L_{\alpha}^{m+1} f(z)\right)^{\prime}+\frac{(1-\alpha) m z\left(R^{m} f(z)\right)^{\prime \prime}}{m+1}\right) \leq F_{h(U)} h(z), \text { i.e., } \\
\left(L_{\alpha}^{m+1} f(z)\right)^{\prime}+\frac{(1-\alpha) m z\left(R^{m} f(z)\right)^{\prime \prime}}{m+1} \prec_{\mathcal{F}} h(z), \quad z \in U,
\end{gathered}
$$

for $\alpha \geq 0$ and $m, n \in \mathbb{N}$, then the fuzzy differential subordination can be written as

$$
F_{L_{\alpha}^{m} f(U)}\left(L_{\alpha}^{m} f(z)\right)^{\prime} \leq F_{q(U)} q(z) \text {, i.e., }\left(L_{\alpha}^{m} f(z)\right)^{\prime} \prec \mathcal{F} q(z), \quad z \in U,
$$

with $q(z)=\frac{1}{n z^{\frac{1}{n}}} \int_{0}^{z} h(t) t^{\frac{1}{n}-1} d t$ being convex and the best fuzzy dominant.

Proof. As $h$ is a convex function of order $-\frac{1}{2}$, Lemma 1 can be applied and we have that $q(z)=\frac{1}{n z^{\frac{1}{n}}} \int_{0}^{z} h(t) t^{\frac{1}{n}-1} d t$ is a convex function and verifies the differential equation associated to the fuzzy differential subordination (19) $q(z)+z q^{\prime}(z)=h(z)$, therefore it is the fuzzy best dominant.

Using the properties of operator $L_{\alpha}^{m}$ and considering $p(z)=\left(L_{\alpha}^{m} f(z)\right)^{\prime}$, we obtain

$$
\left(L_{\alpha}^{m+1} f(z)\right)^{\prime}+\frac{(1-\alpha) m z\left(R^{m} f(z)\right)^{\prime \prime}}{m+1}=p(z)+z p^{\prime}(z), \quad z \in U .
$$

Then, (19) becomes

$$
F_{p(U)}\left(p(z)+z p^{\prime}(z)\right) \leq F_{h(U)} h(z), \quad z \in U .
$$

As $p \in \mathcal{H}[1, n]$, using Lemma 3 , we deduce

$$
F_{p(U)} p(z) \leq F_{q(U)} q(z), \quad z \in U, \quad \text { i.e., } \quad F_{L_{\alpha}^{m} f(U)}\left(L_{\alpha}^{m} f(z)\right)^{\prime} \leq F_{q(U)} q(z), \quad z \in U .
$$

We have obtained

$$
\left(L_{\alpha}^{m} f(z)\right)^{\prime} \prec_{\mathcal{F}} q(z), \quad z \in U .
$$

Corollary 2. Consider the special case when using the convex function $h(z)=\frac{1+(2 \beta-1) z}{1+z}$, where $0 \leq \beta<1$.

If $\alpha \geq 0, m, n \in \mathbb{N}, f \in \mathcal{A}_{n}$ and satisfies the differential subordination

$$
\begin{gathered}
F_{L_{\alpha}^{m} f(U)}\left(\left[L_{\alpha}^{m+1} f(z)\right]^{\prime}+\frac{(1-\alpha) m z\left(R^{m} f(z)\right)^{\prime \prime}}{m+1}\right) \leq F_{h(U)} h(z), \text { i.e. }, \\
{\left[L_{\alpha}^{m+1} f(z)\right]^{\prime}+\frac{(1-\alpha) m z\left(R^{m} f(z)\right)^{\prime \prime}}{m+1} \prec_{\mathcal{F}} h(z), \quad z \in U,}
\end{gathered}
$$


then

$$
F_{L_{\alpha}^{m} f(U)}\left(L_{\alpha}^{m} f(z)\right)^{\prime} \leq F_{q(U)} q(z) \text {, i.e., }\left(L_{\alpha}^{m} f(z)\right)^{\prime} \prec_{\mathcal{F}} q(z), \quad z \in U,
$$

where $q$ is given by $q(z)=2 \beta-1+\frac{2(1-\beta)}{n z^{\frac{1}{n}}} \int_{0}^{z} \frac{t^{\frac{1}{n}-1}}{1+t} d t$, for $z \in U$. The function $q$ is convex and it is the fuzzy best dominant.

Proof. Following the same argumentation as for the proof of Theorem 7 and taking $p(z)=$ $\left(L_{\alpha}^{m} f(z)\right)^{\prime}$, the fuzzy differential subordination (20) becomes

$$
F_{p(U)}\left(p(z)+z p^{\prime}(z)\right) \leq F_{h(U)} h(z), \quad z \in U .
$$

By using Lemma 2 for $\gamma=1$, we have $F_{p(U)} p(z) \leq F_{q(U)} q(z)$, i.e.,

$$
F_{\left(L_{\alpha}^{m} f\right)^{\prime}(U)}\left(L_{\alpha}^{m} f(z)\right)^{\prime} \leq F_{q(U)} q(z) \text {, i.e., }\left(L_{\alpha}^{m} f(z)\right)^{\prime} \prec \mathcal{F} q(z), z \in U,
$$

and $q(z)=\frac{1}{n z^{\frac{1}{n}}} \int_{0}^{z} h(t) t^{\frac{1}{n}-1} d t=\frac{1}{n z^{\frac{1}{n}}} \int_{0}^{z} t^{\frac{1}{n}-1} \frac{1+(2 \beta-1) t}{1+t} d t=2 \beta-1+\frac{2(1-\beta)}{n z^{\frac{1}{n}}} \int_{0}^{z} \frac{t^{\frac{1}{n}}-1}{1+t} d t, z \in$ $U$.

Example 3. Let $h(z)=\frac{1-z}{1+z}$ a convex function in $U$ with $h(0)=1$ and $\operatorname{Re}\left(\frac{z h^{\prime \prime}(z)}{h^{\prime}(z)}+1\right)>-\frac{1}{2}$ (see Example 2).

Let $f(z)=z+z^{2}, z \in U$. For $n=1, m=1$, and $\alpha=2$, we obtain $L_{2}^{1} f(z)=z f^{\prime}(z)=$ $z+2 z^{2}$ and $\left(L_{2}^{1} f(z)\right)^{\prime}=1+4 z$. We also obtain $\left(L_{\alpha}^{m+1} f(z)\right)^{\prime}+\frac{(1-\alpha) m z\left(R^{m} f(z)\right)^{\prime \prime}}{m+1}=\left(L_{2}^{2} f(z)\right)^{\prime}-$ $\frac{z\left(R^{1} f(z)\right)^{\prime \prime}}{2}=\left(z^{2}+z\right)^{\prime}-\frac{z\left(z+2 z^{2}\right)^{\prime \prime}}{2}=1$.

We have $q(z)=\frac{1}{z} \int_{0}^{z} \frac{1-t}{1+t} d t=-1+\frac{2 \ln (1+z)}{z}$.

Using Theorem 8 we obtain

$$
1 \prec \mathcal{F} \frac{1-z}{1+z}, \quad z \in U,
$$

induce

$$
1+4 z \prec \mathcal{F}-1+\frac{2 \ln (1+z)}{z}, \quad z \in U
$$

\section{Conclusions}

Further studies on the newly introduced class can be conducted for obtaining results that give coefficient estimates, distortion theorems, or closure theorems, as it is usual in geometric function theory. Furthermore, the way this class is introduced can inspire research for introducing other interesting fuzzy classes and studying their properties. The limit imposed on $\delta \in(0,1]$ could be further investigated so that other possible values of $\delta$ for correct definitions of fuzzy classes could be found.

Author Contributions: Conceptualization, A.A.L. and G.I.O.; methodology, G.I.O.; software, A.A.L.; validation, A.A.L. and G.I.O.; formal analysis, A.A.L. and G.I.O.; investigation, A.A.L.; resources, G.I.O.; data curation, G.I.O.; writing — original draft preparation, A.A.L.; writing—review and editing, A.A.L. and G.I.O.; visualization, A.A.L.; supervision, G.I.O.; project administration, A.A.L.; funding acquisition, G.I.O. All authors have read and agreed to the published version of the manuscript.

Funding: This research received no external funding.

Institutional Review Board Statement: Not applicable.

Informed Consent Statement: Not applicable.

Data Availability Statement: Not applicable.

Conflicts of Interest: The authors declare no conflict of interest. 


\section{References}

1. Zadeh, L.A. Fuzzy Sets. Inf. Control 1965, 8, 338-353. [CrossRef]

2. Dzitac, I.; Filip, F.G.; Manolescu, M.J. Fuzzy Logic Is Not Fuzzy: World-renowned Computer Scientist Lotfi A. Zadeh. Int. J. Computers Commun. Control. 2017, 12, 748-789. [CrossRef]

3. Dzitac, S.; Nădăban, S. Soft Computing for Decision-Making in Fuzzy Environments: A Tribute to Professor Ioan Dzitac. Mathematics 2021, 9, 1701. [CrossRef]

4. Zadeh, L.A.; Tufis, D.; Filip, F.G.; Dzitac, I. From Natural Language to Soft Computing: New Paradigms in Artificial Intelligence; Editing House of Romanian Academy: Bucharest, Romania, 2008.

5. Oros, G.I.; Oros, G. The notion of subordination in fuzzy sets theory. Gen. Math. 2011, 19, 97-103.

6. Oros, G.I.; Oros, G. Fuzzy differential subordination. Acta Univ. Apulensis 2012, 3, 55-64.

7. Miller, S.S.; Mocanu, P.T. Differential Subordinations. Theory and Applications; Marcel Dekker, Inc.: New York, NY, USA; Basel, Switzerland, 2000.

8. Miller, S.S.; Mocanu, P.T. Second order-differential inequalities in the complex plane. J. Math. Anal. Appl. 1978, 65, 298-305. [CrossRef]

9. Miller, S.S.; Mocanu, P.T. Differential subordinations and univalent functions. Michig. Math. J. 1981, 28, 157-171. [CrossRef]

10. Alb Lupaş, A. A note on special fuzzy differential subordinations using generalized Sălăgean operator and Ruscheweyh derivative. J. Comput. Anal. Appl. 2013, 15, 1476-1483.

11. Alb Lupaş, A.; Oros, G. On special fuzzy differential subordinations using Sălăgean and Ruscheweyh operators. Appl. Math. Comput. 2015, 261, 119-127.

12. Venter, A.O. On special fuzzy differential subordination using Ruscheweyh operator. Analele Univ. Oradea Fasc. Mat. 2015, XXII, 167-176.

13. El-Deeb, S.M.; Alb Lupaş, A. Fuzzy differential subordinations associated with an integral operator. An. Univ. Oradea Fasc. Mat. 2020, XXVII, 133-140.

14. El-Deeb, S.M.; Oros, G.I. Fuzzy differential subordinations connected with the linear operator. Math. Bohem. 2021, 1-10. [CrossRef]

15. Oros, G.I. New fuzzy differential subordinations. Commun. Fac. Sci. Univ. Ank. Ser. A1 Math. Stat. 2021, 70, $229-240$.

16. Srivastava, H.M.; El-Deeb, S.M. Fuzzy Differential Subordinations Based upon the Mittag-Leffler Type Borel Distribution. Symmetry 2021, 13, 1023. [CrossRef]

17. Alb Lupaş, A. On special differential subordinations using Sălăgean and Ruscheweyh operators. Math. Inequal. Appl. 2009, 12, 781-790. [CrossRef]

18. Alb Lupaş, A. On a certain subclass of analytic functions defined by Sălăgean and Ruscheweyh operators. J. Math. Appl. 2009, 31, $67-76$.

19. Gal, S.G.; Ban, A.I. Elemente de Matematică Fuzzy; University of Oradea: Oradea, Romania, 1996.

20. Oros, G.I.; Oros, G. Dominants and best dominants in fuzzy differential subordinations. Stud. Univ. Babeş-Bolyai Math. 2012, 57, 239-248.

21. Sălăgean, G.S. Subclasses of univalent functions. In Lecture Notes in Math; Springer: Berlin, Germany, 1983; Volume 1013; pp. 362-372.

22. Ruscheweyh, S. New criteria for univalent functions. Proc. Amet. Math. Soc. 1975, 49, 109-115. [CrossRef] 